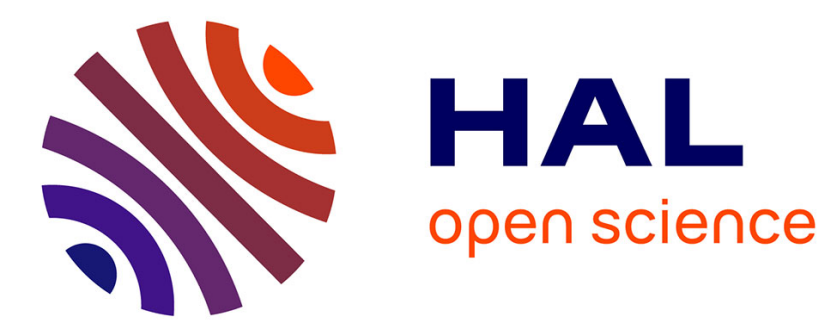

\title{
On the Impact of Transceiver Impairments on AF Relaying
}

Emil Björnson, Agisilaos Papadogiannis, Michail Matthaiou, Mérouane

Debbah

\section{- To cite this version:}

Emil Björnson, Agisilaos Papadogiannis, Michail Matthaiou, Mérouane Debbah. On the Impact of Transceiver Impairments on AF Relaying. ICASSP 2013, May 2013, Vancouver, Canada. pp.4948 4952. hal-00923457

\section{HAL Id: hal-00923457 \\ https://hal-centralesupelec.archives-ouvertes.fr/hal-00923457}

Submitted on 8 Jan 2014

HAL is a multi-disciplinary open access archive for the deposit and dissemination of scientific research documents, whether they are published or not. The documents may come from teaching and research institutions in France or abroad, or from public or private research centers.
L'archive ouverte pluridisciplinaire HAL, est destinée au dépôt et à la diffusion de documents scientifiques de niveau recherche, publiés ou non, émanant des établissements d'enseignement et de recherche français ou étrangers, des laboratoires publics ou privés. 


\title{
ON THE IMPACT OF TRANSCEIVER IMPAIRMENTS ON AF RELAYING
}

\author{
Emil Björnson $^{\star \dagger} \quad$ Agisilaos Papadogiannis ${ }^{\ddagger} \quad$ Michail Matthaiou $^{\ddagger} \quad$ Mérouane Debbah $^{\star}$ \\ ${ }^{\star}$ Alcatel-Lucent Chair on Flexible Radio, SUPELEC, Gif-sur-Yvette, France \\ ${ }^{\dagger}$ ACCESS, Signal Processing Lab, KTH Royal Institute of Technology, Stockholm, Sweden \\ ${ }^{\ddagger}$ Department of Signals and Systems, Chalmers University of Technology, Gothenburg, Sweden \\ Emails: \{emil.bjornson, merouane.debbah\}@ supelec.fr; \{agisilaos.papadogiannis, michail.matthaiou\}@chalmers.se
}

\begin{abstract}
Recently, it was shown that transceiver hardware impairments have a detrimental impact on the performance of communication systems, especially for high-rate systems. The vast majority of technical contributions in the area of relaying assume ideal transceiver hardware. This paper quantifies the impact of transceiver hardware impairments in dual-hop Amplify-and-Forward (AF) relaying, both for fixed and variable gain relays. The outage probability (OP) in this practical scenario is a function of the instantaneous end-toend signal-to-noise-and-distortion ratio (SNDR). This paper derives closed-form expressions for the exact and asymptotic OPs under Rayleigh fading, accounting for hardware impairments at both the transmitter and the relay. The performance loss is small at low spectral efficiency, but can otherwise be very substantial. In particular, it turns out that for high signal-to-noise ratio (SNR), the instantaneous end-to-end SNDR converges to a deterministic constant, called the SNDR ceiling, which is inversely proportional to the level of impairments. This stands in stark contrast to the ideal hardware case for which the end-to-end SNDR grows without bound in the high SNR regime.
\end{abstract}

Index Terms - Transceiver hardware impairments, AF relaying

\section{INTRODUCTION}

The use of relays for improving coverage, throughput, and qualityof-service in wireless systems has been a hot research topic over the past decade, both in academia [1,2] and in industry [3]. This is due to the fact that, unlike base stations, relays are low-cost nodes that can be easily deployed and, hence, enhance the network agility. The vast majority of works in the context of relaying networks make the assumption of ideal transceiver hardware. However, in practice, hardware suffers from impairments; for example, phase noise, IQ imbalance, and amplifier non-linearities [4-6]. These have a deleterious impact on the achievable performance [7-13]. This effect is more pronounced in high-rate systems, especially those employing inexpensive hardware [5]. For instance, some recent works have demonstrated that non-ideal hardware severely affects single-hop multiantenna systems; $[6,8]$ proved that there is a finite capacity limit at high SNR, while $[9,13]$ showed that existing signal processing algorithms need to be re-designed to account for these impairments.

Despite the importance of transceiver impairments, their impact on relaying has only been partially investigated; in particular, bit

E. Björnson is funded by the International Postdoc Grant 2012-228 from The Swedish Research Council. This research has been supported by the ERC Starting Grant 305123 MORE (Advanced Mathematical Tools for Complex Network Engineering).

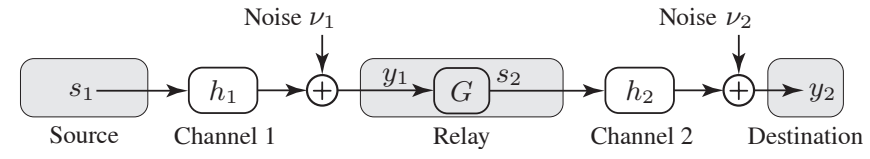

(a) Classical system model with ideal transceiver hardware.

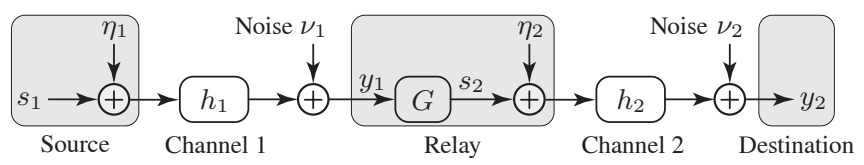

(b) Generalized system model with transceiver impairments.

Fig. 1: Block diagram of AF relaying with (a) ideal hardware or (b) transceiver impairments with aggregate distortion noise $\eta_{i}, i=1,2$.

error rate simulations were conducted in [10], while [11, 12] derived expressions for the bit/symbol error rates, considering only non-linearities or IQ imbalance, respectively. In this paper, we try to bridge this gap by analytically evaluating the performance of dualhop AF relaying networks in the presence of aggregate transceiver impairments, both for fixed and variable gain relaying. In particular, we obtain the SNDRs and tractable closed-form expressions for the exact and asymptotic OPs of the system. This enables us to characterize the impact of impairments. To the best of our knowledge, this is the first paper presenting an analytical study of relaying with transceiver impairments under the generalized system model of [5].

One important observation is that for both types of relaying, an increase in the SNRs lead to an "SNDR ceiling" effect, which is explicitly quantified. The value of the SNDR ceiling is inversely proportional to the level of impairments. This manifests that transceiver impairments have a significant impact on AF relaying — especially at high SNRs and when high spectral efficiencies are desirable.

\section{SIGNAL AND SYSTEM MODEL}

This paper revisits the classical dual-hop relaying in Fig. 1a, where a source communicates with a destination through a relay using AF.

\subsection{Ideal Hardware}

In the classical complex baseband system model (see Fig. 1a), the received signals at the relay and destination are

$$
y_{i}=h_{i} s_{i}+\nu_{i}, \quad i=1,2,
$$

where $s_{1}, s_{2} \in \mathbb{C}$ are the transmitted signals from the source and relay, respectively, with average signal power $P_{i}=\mathbb{E}_{s_{i}}\left\{\left|s_{i}\right|^{2}\right\}$, for 
$i=1,2$. Observe that $\mathbb{E}\{\cdot\}$ denotes the expectation operator. In addition, $\nu_{i} \sim \mathcal{C N}\left(0, N_{i}\right)$ represents the additive circularly-symmetric complex Gaussian noise terms for $i=1,2$. The random channel fading coefficients are modeled as Rayleigh fading ${ }^{1}$ such that $h_{i} \sim \mathcal{C N}\left(0, \Omega_{i}\right)$, where $\Omega_{i} \triangleq \mathbb{E}_{h_{i}}\left\{\left|h_{i}\right|^{2}\right\}$ is the average power of the fading channel. Recall that, in this case, the cumulative distribution functions of the channel gains, $\rho_{i}=\left|h_{i}\right|^{2}$, are given by

$$
F_{\rho_{i}}(x)=1-e^{-\frac{x}{\Omega_{i}}}, \quad x \geq 0, \quad i=1,2 .
$$

The quantity $\frac{P_{i} \Omega_{i}}{N_{i}}$ will be referred to as the average $S N R$, for $i=$ 1,2. A high SNR is achieved by high signal and/or fading power.

The transmitted signal $s_{2}$ at the relay is simply an amplified version of the signal $y_{1}$ received at the relay: $s_{2}=G y_{1}$. If the relay has instantaneous knowledge of the fading channel, $h_{1}$, it can apply variable gain relaying with $G^{\mathrm{v}} \triangleq \sqrt{P_{2} / \mathbb{E}_{s_{1}, \nu_{1}}\left\{\left|y_{1}\right|^{2}\right\}}$ [14]. Otherwise, fixed gain relaying with $G^{\mathrm{f}} \triangleq \sqrt{P_{2} / \mathbb{E}_{s_{1}, \nu_{1}, h_{1}}\left\{\left|y_{1}\right|^{2}\right\}}$ can be applied using only statistical information [2]. ${ }^{2}$ With ideal (id) hardware, the amplification factors can be expressed as

$$
G_{\mathrm{id}}^{\mathrm{f}}=\sqrt{\frac{P_{2}}{P_{1} \Omega_{1}+N_{1}}}, \quad G_{\mathrm{id}}^{\mathrm{v}}=\sqrt{\frac{P_{2}}{P_{1} \rho_{1}+N_{1}}} .
$$

The received signal at the destination is

$$
y_{2}=h_{2} G_{\text {id }}\left(h_{1} s_{1}+\nu_{1}\right)+\nu_{2} .
$$

After some simple algebraic manipulations, the end-to-end SNDRs of (1) for variable and fixed gain relays are obtained as

$$
\gamma_{\mathrm{id}}^{\mathrm{f}}=\frac{P_{1} \rho_{1} \rho_{2}}{\rho_{2} N_{1}+N_{2} /\left(G_{\mathrm{id}}^{\mathrm{f}}\right)^{2}}, \quad \gamma_{\mathrm{id}}^{\mathrm{v}}=\frac{P_{1} P_{2} \rho_{1} \rho_{2}}{P_{2} \rho_{2} N_{1}+\left(P_{1} \rho_{1}+N_{1}\right) N_{2}},
$$

respectively. Similar expressions were previously derived in $[2,14]$.

\subsection{Non-Ideal Hardware: Transceiver Impairments}

The model in (1) implicitly assumes ideal transceiver hardware. However, physical radio-frequency (RF) transceivers suffer from a variety of impairments that create a mismatch between the intended signal for transmission, $s_{i}$, and the signal that is actually generated and emitted. The impact of each impairment (e.g., phase-noise, IQ imbalance, and amplifier non-linearities) can be modeled separately for a given hardware setup $[5,10]$. Interestingly, the combined influence is well-modeled by the generalized system model

$$
y_{i}=h_{i}\left(s_{i}+\eta_{i}^{\mathrm{tx}}\right)+\eta_{i}^{\mathrm{rx}}+\nu_{i}, \quad i=1,2,
$$

where $\eta_{i}^{\mathrm{tx}}, \eta_{i}^{\mathrm{rx}}$ are distortion noises in the transmitter and the receiver, respectively [5]. Experiments and theoretical investigations (e.g., $[4,6,7])$ have verified that these are well-modeled as

$$
\eta_{i}^{\mathrm{tx}} \sim \mathcal{C N}\left(0,\left(\kappa_{i}^{\mathrm{tx}}\right)^{2} \mathcal{P}\right), \quad \eta_{i}^{\mathrm{rx}} \sim \mathcal{C} \mathcal{N}\left(0,\left(\kappa_{i}^{\mathrm{rx}}\right)^{2}\left|h_{i}\right|^{2} \mathcal{P}\right)
$$

where the Gaussianity can be explained by the aggregate effect of many impairments. ${ }^{3}$ Note that the distortion depends on the intended signal for transmission/reception, in the sense that the variance of $\eta_{i}$ is proportional to its average power, here denoted as $\mathcal{P}$.

For convenience, we define $\eta_{i} \triangleq \eta_{i}^{\mathrm{tx}}+\eta_{i}^{\mathrm{rx}} / h_{i} \sim \mathcal{C N}\left(0, \kappa_{i}^{2} \mathcal{P}\right)$ as the aggregate distortion noise, where $\kappa_{i}^{2}=\left(\kappa_{i}^{\mathrm{tx}}\right)^{2}+\left(\kappa_{i}^{\mathrm{rx}}\right)^{2}$. The

\footnotetext{
${ }^{1}$ Note that the following analysis is generic and applies for any fading distribution. We focus on the case of Rayleigh fading for the sake of tractability.

${ }^{2}$ The relay then has a long-term power constraint $P_{2}=\mathbb{E}\left\{\left|G_{\text {id }}^{\mathrm{f}} y_{1}\right|^{2}\right\}$ where expectation is taken over signal, noise and channel fading realizations.

${ }^{3}$ The Gaussianity holds in particular for the residual distortion when compensation algorithms are applied to handle multiplicative signal errors [6].
}

parameter $\kappa_{i}>0$ describes the level of impairments on the $i$ th channel and is an important parameter in this paper. Note that $\kappa_{i}$ can be interpreted as the total error vector magnitude (EVM) - a common measure for quantifying the quality of RF transceivers. ${ }^{4}$ This parameter is essentially constant while operating in the dynamic range of the power amplifier, but can otherwise increase very rapidly [7,9]. The generalized system model of (6) is illustrated in Fig. 1b.

For the non-ideal (ni) hardware counterpart of (4), the received signal at the destination is now obtained as

$$
\begin{aligned}
y_{2} & =h_{2} G_{\mathrm{ni}}\left(h_{1}\left(s_{1}+\eta_{1}\right)+\nu_{1}\right)+h_{2} \eta_{2}+\nu_{2} \\
& =G_{\mathrm{ni}} h_{1} h_{2} s_{1}+G_{\mathrm{ni}} h_{1} h_{2} \eta_{1}+G_{\mathrm{ni}} h_{2} \nu_{1}+h_{2} \eta_{2}+\nu_{2} .
\end{aligned}
$$

For fixed and variable gain relaying, $G_{\mathrm{ni}}$ reads respectively as

$$
G_{\mathrm{ni}}^{\mathrm{f}} \triangleq \sqrt{\frac{P_{2}}{P_{1} \Omega_{1}\left(1+\kappa_{1}^{2}\right)+N_{1}}}, G_{\mathrm{ni}}^{\mathrm{v}} \triangleq \sqrt{\frac{P_{2}}{P_{1} \rho_{1}\left(1+\kappa_{1}^{2}\right)+N_{1}}} .
$$

The aggregate distortion noise is equal to $\eta_{1} \sim \mathcal{C N}\left(0, \kappa_{1}^{2} P_{1}\right)$ for the source and $\eta_{2} \sim \mathcal{C N}\left(0, \kappa_{2}^{2} G_{\mathrm{ni}}^{2} \mathbb{E}_{s_{1}, \nu_{1}}\left\{\left|y_{1}\right|^{2}\right\}\right)$ for the relay. The latter becomes $\eta_{2}^{\mathrm{f}} \sim \mathcal{C N}\left(0,\left(G_{\mathrm{ni}}^{\mathrm{f}}\right)^{2} \kappa_{2}^{2}\left(P_{1} \rho_{1}\left(1+\kappa_{1}^{2}\right)+N_{1}\right)\right)$ and $\eta_{2}^{\mathrm{v}} \sim \mathcal{C N}\left(0, \kappa_{2}^{2} P_{2}\right)$ for fixed and variable gain relays, respectively. After some algebraic manipulations (using the expressions for $G_{\mathrm{ni}}^{\mathrm{v}}$ ), the end-to-end SNDRs for fixed and variable gain relaying are

$$
\begin{aligned}
\gamma_{\mathrm{ni}}^{\mathrm{f}} & =\frac{P_{1} \rho_{1} \rho_{2}}{P_{1} \rho_{1} \rho_{2} d+\rho_{2} N_{1}\left(1+\kappa_{2}^{2}\right)+\frac{N_{2}}{\left(G_{\mathrm{ni}}^{\mathrm{f}}\right)^{2}}}, \\
\gamma_{\mathrm{ni}}^{\mathrm{v}} & =\frac{P_{1} P_{2} \rho_{1} \rho_{2}}{P_{1} P_{2} \rho_{1} \rho_{2} d+\rho_{1} P_{1} N_{2}\left(1+\kappa_{1}^{2}\right)+\rho_{2} P_{2} N_{1}\left(1+\kappa_{2}^{2}\right)+N_{1} N_{2}},
\end{aligned}
$$

respectively, where $d \triangleq \kappa_{1}^{2}+\kappa_{2}^{2}+\kappa_{1}^{2} \kappa_{2}^{2}$ will appear repeatedly in the paper. These SNDR expressions reduce to (5) for $\kappa_{1}=\kappa_{2}=0$.

\section{OUTAGE PROBABILITY ANALYSIS}

This section derives new analytical expressions for the exact and asymptotic OPs with AF under the presence of transceiver impairments, for fixed and variable gain relaying. These results are compared with the standard results in the literature based on the assumption of ideal hardware. The OP, $P_{\text {out }}(x)$, is defined as the probability that the channel fading makes the SNDR fall below a certain threshold $x$; that is,

$$
P_{\text {out }}(x)=\operatorname{Pr}\{\gamma \leq x\},
$$

where $\gamma$ is the end-to-end SNDR of the system.

\subsection{Exact SNR Analysis: Arbitrary Fading Distributions}

This subsection considers the OP with arbitrary channel fading. The following lemma plays an important role when deriving $P_{\text {out }}(x)$.

Lemma 1. Let $c_{1}, c_{2}, c_{3}$ be strictly positive constants and let $\rho$ be a non-negative random variable with cdf $F_{\rho}(\cdot)$. Then,

$$
\operatorname{Pr}\left\{\frac{c_{1} \rho}{c_{2} \rho+c_{3}} \leq x\right\}= \begin{cases}F_{\rho}\left(\frac{c_{3} x}{c_{1}-c_{2} x}\right), & 0 \leq x<\frac{c_{1}}{c_{2}}, \\ 1, & x \geq \frac{c_{1}}{c_{2}} .\end{cases}
$$

Suppose $c_{2}=0$ instead, then (13) simplifies to

$$
\operatorname{Pr}\left\{\frac{c_{1} \rho}{c_{3}} \leq x\right\}=F_{\rho}\left(\frac{c_{3} x}{c_{1}}\right) .
$$

${ }^{4}$ The total EVM is defined as $\sqrt{\mathbb{E}_{\eta_{i}}\left\{\left|\eta_{i}\right|^{2}\right\} / \mathbb{E}_{s_{i}}\left\{\left|s_{i}\right|^{2}\right\}}$. 3GPP LTE has EVM requirements for transmitters in the range $\kappa_{i}^{\text {tx }} \in[0.08,0.175]$, where smaller values are needed to support high spectral efficiency [15, Sec. 14.3.4]. 
Proof. The left-hand side of (13) is equal to

$$
\operatorname{Pr}\left\{c_{1} \rho \leq\left(c_{2} \rho+c_{3}\right) x\right\}=\operatorname{Pr}\left\{\rho \leq \frac{c_{3} x}{\left(c_{1}-c_{2} x\right)}\right\}
$$

after some algebra. The last expression is exactly $F_{\rho}\left(\frac{c_{3} x}{c_{1}-c_{2} x}\right)$. Note that if $\left(c_{1}-c_{2} x\right) \leq 0$, then the outage event always occurs.

The effective SNDRs under AF relaying are given in (5) and (10)-(11) for ideal and non-ideal transceiver hardware, respectively. The OPs can be computed using Lemma 1.

Theorem 1. Suppose $\rho_{i}$ is an independent non-negative random variable with $c d f F_{\rho_{i}}(\cdot)$ and $p d f f_{\rho_{i}}(\cdot)$ for $i=1,2$. For fixed and variable gain relaying, the OPs with ideal hardware are

$$
\begin{aligned}
& P_{\text {out }}^{\text {id,f }}(x)=\int_{0}^{\infty} F_{\rho_{1}}\left(\frac{x\left(N_{1} \rho_{2}+\frac{N_{2}}{\left(G_{\mathrm{id}}^{\mathrm{f}}\right)^{2}}\right)}{P_{1} \rho_{2}}\right) f_{\rho_{2}}\left(\rho_{2}\right) d \rho_{2} \\
& P_{\text {out }}^{\text {id, }}(x)=1-\int_{0}^{\infty}\left(1-F_{\rho_{1}}\left(\frac{\left(x+x^{2}\right)+\frac{P_{2} x}{N_{2}} z}{\frac{P_{1} P_{2}}{N_{1} N_{2}} z}\right)\right) f_{\rho_{2}}\left(z+\frac{N_{2} x}{P_{2}}\right) d z,
\end{aligned}
$$

respectively. The corresponding OPs with transceiver hardware impairments satisfy $P_{\mathrm{out}}^{\mathrm{ni}, \mathrm{f}}(x)=P_{\mathrm{out}}^{\mathrm{ni}, \mathrm{v}}(x)=1$ for $x \geq \frac{1}{d}$ and

$$
\begin{aligned}
& P_{\text {out }}^{\mathrm{ni}, \mathrm{f}}(x)=\int_{0}^{\infty} F_{\rho_{1}}\left(\frac{\left(b_{1} \rho_{2}+a_{1}\right) x}{\rho_{2}(1-x d)}\right) f_{\rho_{2}}\left(\rho_{2}\right) d \rho_{2} \\
& P_{\text {out }}^{\mathrm{ni}, \mathrm{v}}(x)=1-\int_{0}^{\infty}\left(1-F_{\rho_{1}}\left(\frac{\frac{x+x^{2}}{1-x d}+\frac{b_{1} x z}{a_{2}}}{\frac{(1-x d)}{a_{2}} z}\right)\right) f_{\rho_{2}}\left(z+\frac{x b_{2}}{1-x d}\right) d z,
\end{aligned}
$$

for $x<\frac{1}{d}$. In these expressions, we have $a_{1} \triangleq \frac{N_{2}}{P_{1}\left(G_{\mathrm{ni}}^{\mathrm{f}}\right)^{2}}, a_{2} \triangleq$ $\frac{N_{1} N_{2}}{P_{1} P_{2}}, b_{1} \triangleq \frac{N_{1}\left(1+\kappa_{2}^{2}\right)}{P_{1}}$, and $b_{2} \triangleq \frac{N_{2}\left(1+\kappa_{1}^{2}\right)}{P_{2}}$.

Proof. The OP in (12) depends on the end-to-end SNDR $\gamma$, which is a function of both $\rho_{1}$ and $\rho_{2}$. Using the law of total probability to condition on $\rho_{2}$, we can write $\operatorname{Pr}\{\gamma \leq x\}=\int_{0}^{\infty} \operatorname{Pr}\{\gamma \leq$ $\left.x \mid \rho_{2}\right\} f_{\rho_{2}}\left(\rho_{2}\right) d \rho_{2}$. The conditional probability $\operatorname{Pr}\left\{\gamma \leq x \mid \rho_{2}\right\}$ can then be evaluated using Lemma 1, which immediately gives (16) and (18). Finally, (17) is obtained by the additional change of variable $z=\rho_{2}-\frac{N_{2} x}{P_{2}}$, while (19) is achieved by setting $z=\rho_{2}-\frac{x b_{2}}{1-x d}$.

This theorem provides integral expressions for the OPs. In the following, we particularize these expressions to the case of Rayleigh fading. Interestingly, the OP with transceiver impairments always equals 1 when $x \geq \frac{1}{d}$. This has an intuitive explanation since $\gamma \leq \frac{1}{d}$ for any transmit power and fading distribution; see Section 3.3.

\subsection{Exact SNR Analysis: Rayleigh Fading Channels}

Under Rayleigh fading and ideal hardware, the OPs with fixed and variable gain relaying were obtained in [2, Eq. (9)] and [14, Eq. (14)], respectively. Using our notation, we get

$$
\begin{aligned}
& P_{\text {out }}^{\mathrm{id}, \mathrm{f}}(x)=1-2 e^{-\frac{N_{1} x}{P_{1} \Omega_{1}}} \sqrt{c_{\mathrm{id}}^{\mathrm{f}} x} K_{1}\left(2 \sqrt{c_{\mathrm{id}}^{\mathrm{f}} x}\right) \\
& P_{\text {out }}^{\mathrm{id}, \mathrm{v}}(x)=1-2 e^{-x\left(\frac{N_{1}}{P_{1} \Omega_{1}}+\frac{N_{2}}{P_{2} \Omega_{2}}\right)} \sqrt{\frac{x+x^{2}}{c_{\mathrm{id}}^{\mathrm{v}}}} K_{1}\left(2 \sqrt{\frac{x+x^{2}}{c_{\mathrm{id}}^{\mathrm{v}}}}\right),
\end{aligned}
$$

where $c_{\mathrm{id}}^{\mathrm{f}} \triangleq \frac{N_{2}}{P_{1}\left(G_{\mathrm{id}}^{\mathrm{f}}\right)^{2} \Omega_{1} \Omega_{2}}, c_{\mathrm{id}}^{\mathrm{v}} \triangleq \frac{P_{1} P_{2} \Omega_{1} \Omega_{2}}{N_{1} N_{2}}$, and $K_{1}(\cdot)$ denotes the first-order modified Bessel function of the second kind.

The non-ideal case with transceiver hardware impairments is more involved, as can be seen from the following corollary.

Corollary 1. Suppose $h_{1}, h_{2}$ are independent and $h_{i} \sim \mathcal{C N}\left(0, \Omega_{i}\right)$ for $i=1,2$. The OPs under transceiver hardware impairments are

$P_{\text {out }}^{\mathrm{ni}, \mathrm{f}}(x)= \begin{cases}1-2 e^{-\frac{B_{\mathrm{ni}}^{\mathrm{f}} x}{1-x d}} \sqrt{\frac{A_{\mathrm{n} i}^{\mathrm{f}} x}{1-x d}} K_{1}\left(2 \sqrt{\frac{A_{\mathrm{ni}}^{\mathrm{f} x}}{1-x d}}\right), & x<\frac{1}{d}, \\ 1, & x \geq \frac{1}{d},\end{cases}$
$P_{\text {out }}^{\mathrm{ni}, \mathrm{v}}(x)= \begin{cases}1-2 e^{-\frac{B_{\mathrm{ni}}^{\mathrm{v}} x}{1-x d}} \frac{\sqrt{A_{\mathrm{ni}}^{\mathrm{v}}\left(x+x^{2}\right)}}{1-x d} K_{1}\left(2 \frac{\sqrt{A_{\mathrm{ni}}^{\mathrm{v}}\left(x+x^{2}\right)}}{1-x d}\right), & x<\frac{1}{d}, \\ 1, & x \geq \frac{1}{d},\end{cases}$

for fixed and variable gain relaying, respectively. In these expressions, we have $A_{\mathrm{ni}}^{\mathrm{f}} \triangleq \frac{N_{2}}{P_{1}\left(G_{\mathrm{ni}}^{\mathrm{f}}\right)^{2} \Omega_{1} \Omega_{2}}, B_{\mathrm{ni}}^{\mathrm{f}} \triangleq \frac{N_{1}\left(1+\kappa_{2}^{2}\right)}{\Omega_{1} P_{1}}, A_{\mathrm{ni}}^{\mathrm{v}} \triangleq$ $\frac{N_{1} N_{2}}{P_{1} P_{2} \Omega_{1} \Omega_{2}}$, and $B_{\mathrm{ni}}^{\mathrm{v}} \triangleq \frac{N_{1}\left(1+\kappa_{2}^{2}\right)}{\Omega_{1} P_{1}}+\frac{N_{2}\left(1+\kappa_{1}^{2}\right)}{\Omega_{2} P_{2}}$.

Proof. The proof consists of evaluating (18) and (19) using the fading distribution in (2). For fixed gain relaying, $P_{\text {out }}^{\text {ni,f }}(x)$ becomes

$$
\begin{aligned}
& \int_{0}^{\infty}\left(1-e^{-\frac{\left(b_{1} \rho_{2}+a_{1}\right) x}{\Omega_{1} \rho_{2}(1-x d)}}\right) e^{-\frac{\rho_{2}}{\Omega_{2}} d \rho_{2}} \\
& \quad=1-e^{-\frac{B_{\mathrm{ni}}^{\mathrm{f}} x}{1-x d}} \int_{0}^{\infty} e^{-\frac{A_{\mathrm{ni}}^{\mathrm{f}} x}{\rho_{2}(1-x d)}} e^{-\frac{\rho_{2}}{\Omega_{2}}} d \rho_{2}
\end{aligned}
$$

for $x<\frac{1}{d}$ and is equal to 1 otherwise. The remaining integral is evaluated using [16, Eq. (3.324.1)], leading to (22). The expression for $P_{\text {out }}^{\mathrm{ni}, \mathrm{v}}(x)$ is obtained in the same way.

This corollary provides new and tractable closed-form expressions for the OPs in case of transceiver impairments.

\subsection{Asymptotic SNR Analysis}

To obtain some insights on the fundamental impact of impairments, we now elaborate on the high-SNR regime. For the ease of presentation, we assume that $P_{1}, P_{2}$ grow large with $P_{1}=\beta P_{2}$ for some $\beta>0$, such that the relay gain remains finite and strictly positive.

Corollary 2. Suppose $P_{1}, P_{2}$ grow large with a finite non-zero ratio and consider any random distributions on $\rho_{1}, \rho_{2}$ that are strictly positive (with probability one). The asymptotic OP with transceiver impairments and either fixed or variable gain relaying satisfies

$$
\lim _{P_{1}, P_{2} \rightarrow \infty} P_{\text {out }}(x)= \begin{cases}0, & x \leq \frac{1}{\kappa_{1}^{2}+\kappa_{2}^{2}+\kappa_{1}^{2} \kappa_{2}^{2}}, \\ 1, & x>\frac{1}{\kappa_{1}^{2}+\kappa_{2}^{2}+\kappa_{1}^{2} \kappa_{2}^{2}} .\end{cases}
$$

Proof. Referring back to (10) and by taking the limit $P_{1}, P_{2} \rightarrow \infty$ (with $P_{1}=\beta P_{2}$ ), we can easily see that the instantaneous end-toend SNDR, for fixed gain relaying, converges to

$$
\lim _{P_{1}, P_{2} \rightarrow \infty} \gamma_{\mathrm{ni}}^{\mathrm{f}}=\gamma^{*}=\frac{1}{d}=\frac{1}{\kappa_{1}^{2}+\kappa_{2}^{2}+\kappa_{1}^{2} \kappa_{2}^{2}} .
$$

Therefore, (25) is trivially obtained. The proof for the case of variable gain relaying follows a similar line of reasoning.

A number of conclusions can be drawn from Corollary 2. First, an SNDR ceiling effect appears in the high-SNR regime, which significantly limits the performance of AF relaying systems. This phenomenon is fundamentally different from the ideal hardware case, in 


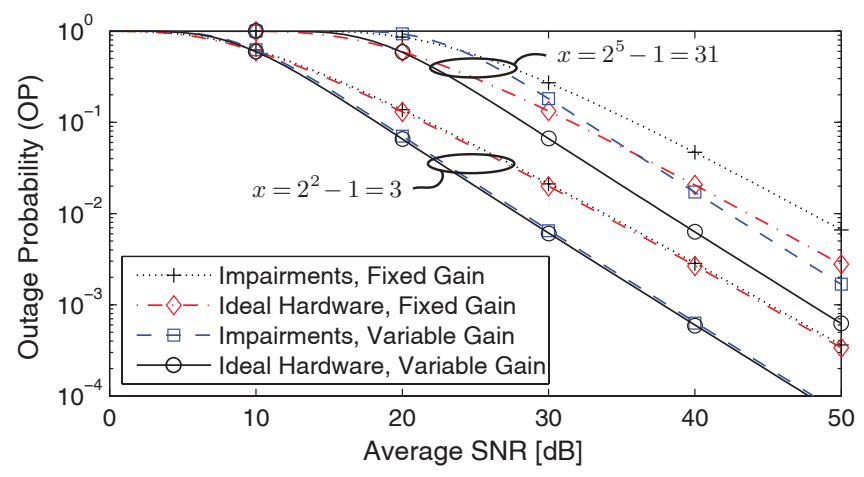

Fig. 2: Outage probability $P_{\text {out }}(x)$ with $\mathrm{AF}$ relaying with ideal and non-ideal transceiver hardware.

which an increasing SNR makes the end-to-end SNDR grow without bound and $P_{\text {out }}(x) \rightarrow 0$ for any $x$. Note that this ceiling effect is independent of the fading distributions of the two hops.

Moreover, the SNDR ceiling $\gamma^{*}$ in (26) is inversely proportional to the squares of $\kappa_{1}, \kappa_{2}$. We also note that the impact of $\kappa_{1}$ and $\kappa_{2}$ on the value of $\gamma^{*}$ is symmetric. Recall that these parameters describe the level of transceiver impairments and equal the EVMs of the source and relay hardware, respectively. This validates that transceiver impairments dramatically affect performance of AF relaying, and, hence, they should be taken into account when designing and evaluating relaying networks. To this end, we now assume that we have a fixed total EVM constraint $\kappa_{\text {tot }}$, such that $\kappa_{1}+\kappa_{2}=\kappa_{\text {tot }}$, and present the following insightful corollary.

Corollary 3. Suppose $\kappa_{1}+\kappa_{2}=\kappa_{\text {tot }}$ for some constant $0<\kappa_{\text {tot }}<$ 2. The SNDR ceiling in (26) is maximized if $\kappa_{1}=\kappa_{2}=\frac{\kappa_{\mathrm{tot}}}{2}$.

Proof. The maximization of (26) is equivalent to a minimization of $f\left(\kappa_{1}\right)=\kappa_{1}^{2}+\left(\kappa_{\text {tot }}-\kappa_{1}\right)^{2}+\kappa_{1}^{2}\left(\kappa_{\text {tot }}-\kappa_{1}\right)^{2}$. Simple differentiation reveals that the function is convex for any $0<\kappa_{\text {tot }}<2$ and that $\kappa_{1}=\frac{\kappa_{\text {tot }}}{2}$ is the only real-valued solution to $\frac{\partial}{\partial \kappa_{1}} f\left(\kappa_{1}\right)=0$.

Corollary 3 shows that it is better to have the same hardware quality on the two hops of the relay channel, than having one highquality node and one low-quality node. We finally point out that the SNDR ceiling can be trivially bounded as $\frac{1}{\left(\kappa_{1}+\kappa_{2}\right)^{2}} \leq \gamma^{*} \leq \frac{1}{\kappa_{1}^{2}+\kappa_{2}^{2}}$.

\section{NUMERICAL ILLUSTRATIONS}

This section provides numerical illustrations of our analytical results. We consider a symmetric Rayleigh fading scenario where the average $\mathrm{SNR}, \frac{P_{i} \Omega_{i}}{N_{i}}$, is the same for $i=1,2$. This enables us to focus on the impact of transceiver hardware impairments.

The OP, $P_{\text {out }}(x)$, with fixed and variable gain relaying is shown in Fig. 2 as a function of the average SNR. We compare the system performance with ideal transceivers and non-ideal transceivers with $\kappa_{1}=\kappa_{2}=0.1$. Two thresholds are considered: $x=2^{2}-1=3$ and $x=2^{5}-1=31$. These correspond to spectral efficiencies of 2 and $5 \mathrm{bits} / \mathrm{s} / \mathrm{Hz}$, respectively. The curves were generated by the analytical expressions in (20)-(23), while the marker symbols show the results of Monte-Carlo simulations. As seen in Fig. 2, there is a minor performance loss caused by transceiver impairments in the low threshold case of $x=3$. However, there is a substantial performance loss of around $5 \mathrm{~dB}$ in SNR when the threshold is increased to $x=31$. This effect applies to both variable and fixed gain relaying. Note that variable gain relaying outperforms the fixed one in most scenarios of interest, which is in line with [2].

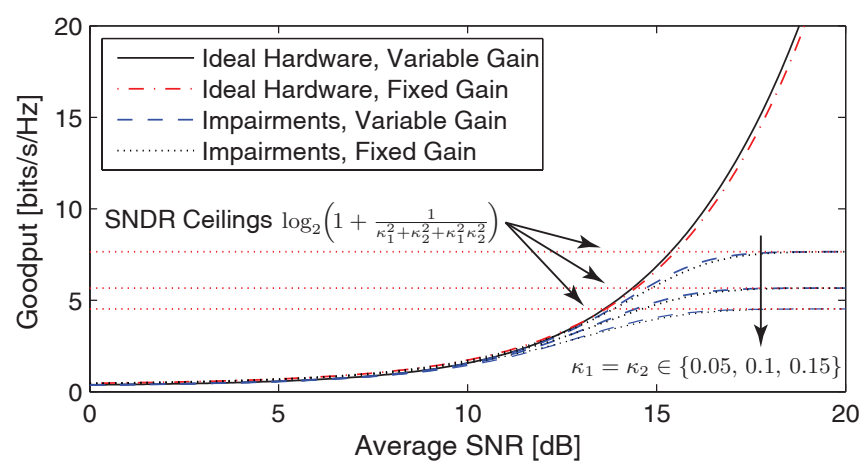

Fig. 3: Maximum goodput $R_{\text {goodput }}(x)$ with $\mathrm{AF}$ relaying with ideal and non-ideal transceiver hardware.

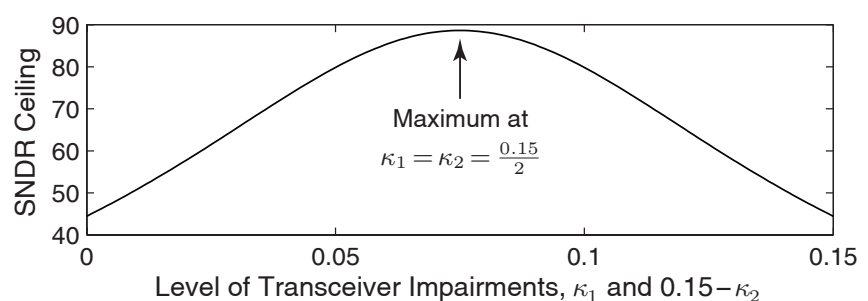

Fig. 4: Value of the SDNR ceiling in (26) for different levels of impairments $\kappa_{1}, \kappa_{2}$ for which $\kappa_{1}+\kappa_{2}=0.15$.

Next, we consider the average rate of data correctly received under fading (in bits/s/Hz) [17, Chapter 4.2.3], or the so-called goodput [18]. This is defined as the product between the gross spectral efficiency and the corresponding probability of successful communication. For any given setup, the maximum goodput is defined as

$$
R_{\text {goodput }}=\max _{x \geq 0} \log _{2}(1+x)\left(1-P_{\text {out }}(x)\right) .
$$

The analytical expressions for $P_{\text {out }}(x)$ in (20)-(23) allow simple numerical computation of the maximum goodput in (27). This is illustrated in Fig. 3 for different levels of impairments: $\kappa_{1}=\kappa_{2} \in$ $\{0.05,0.1,0.15\}$. This figure demonstrates the deleterious impact of non-ideal hardware; the goodput with transceiver impairments saturates at high SNR and approaches $\log _{2}\left(1+\gamma^{*}\right)$, where $\gamma^{*}=$ $\frac{1}{\kappa_{1}^{2}+\kappa_{2}^{2}+\kappa_{1}^{2} \kappa_{2}^{2}}$ is the SNDR ceiling. This is consistent with Corollary 2, because $\gamma^{*}$ is the highest SNDR for which the OP approaches zero as $P_{1}, P_{2} \rightarrow \infty$. The saturation level increases when $\kappa_{1}, \kappa_{2}$ are decreased. Finally, Fig. 4 shows the value of the SNDR ceiling for different sets of $\kappa_{1}, \kappa_{2}$ for which $\kappa_{1}+\kappa_{2}=0.15$. As stated in Corollary 3 , the maximum is achieved by $\kappa_{i}=\frac{0.15}{2}$ for $i=1,2$.

\section{CONCLUSIONS}

Physical transceiver hardware introduces impairments that distort the emitted signals. Despite the practical importance of these impairments, little was known about their impact on the achievable performance of relaying systems. This paper has analytically shown that the performance of dual-hop AF relaying is highly affected by these impairments, particularly when high spectral efficiencies are required. Closed-form expressions for the exact and asymptotic OPs were derived for fixed and variable gain relaying. These expressions effectively characterize the impact of impairments and manifest the existence of an SNDR ceiling that cannot be crossed by increasing transmit powers or improving fading conditions. The engineering insight is that both hops should have the same hardware quality. 


\section{REFERENCES}

[1] J. N. Laneman, D. N. C. Tse, and G. W. Wornell, "Cooperative diversity in wireless networks: Efficient protocols and outage behavior," IEEE Trans. Inf. Theory, vol. 50, no. 12, pp. 30623080, Dec. 2004.

[2] M. O. Hasna and M.-S. Alouini, "A performance study of dualhop transmissions with fixed gain relays," IEEE Trans. Wireless Commun., vol. 3, no. 6, pp. 1963-1968, Nov. 2004.

[3] Y. Hua, D. W. Bliss, S. Gazor, Y. Rong, and Y. Sung, "Theories and methods for advanced wireless relays-Issue I," IEEE J. Sel. Areas Commun., vol. 30, no. 8, pp. 1297-1303, Sept. 2012.

[4] E. Costa and S. Pupolin, " $m$-QAM-OFDM system performance in the presence of a nonlinear amplifier and phase noise," IEEE Trans. Commun., vol. 50, no. 3, pp. 462-472, Mar. 2002.

[5] T. Schenk, RF Imperfections in High-Rate Wireless Systems: Impact and Digital Compensation, Springer, 2008.

[6] C. Studer, M. Wenk, and A. Burg, "MIMO transmission with residual transmit-RF impairments," in Proc. ITG/IEEE Workshop on Smart Antennas (WSA), Feb. 2010.

[7] P. Zetterberg, "Experimental investigation of TDD reciprocitybased zero-forcing transmit precoding," EURASIP J. on Adv. in Signal Process., Jan. 2011.

[8] E. Björnson, P. Zetterberg, M. Bengtsson, and B. Ottersten, "Capacity limits and multiplexing gains of MIMO channels with transceiver impairments," IEEE Commun. Lett., vol. 17, no. 1, pp. 91-94, Jan. 2013.

[9] E. Björnson, P. Zetterberg, and M. Bengtsson, "Optimal coordinated beamforming in the multicell downlink with transceiver impairments," in Proc. IEEE GLOBECOM, Dec. 2012.

[10] J. Samuel, P. Rosson, L. Maret, C. Dehos, and A. Valkanas, "Impact of RF impairments in cellular wireless metropolitan area networks," in Proc. IEEE ISSSTA, Aug. 2008.

[11] T. Riihonen, S. Werner, F. Gregorio, R. Wichman, and J. Hämäläinen, "BEP analysis of OFDM relay links with nonlinear power amplifiers," in Proc. IEEE WCNC, Apr. 2010.

[12] J. Qi, S. Aïssa, and M.-S. Alouini, "Analysis and compensation of I/Q imbalance in amplify-and-forward cooperative systems," in Proc. IEEE WCNC, Apr. 2012, pp. 215-220.

[13] E. Björnson and E. Jorswieck, "Optimal resource allocation in coordinated multi-cell systems," Foundations and Trends in Communications and Information Theory, vol. 9, no. 2-3, pp. 113-381, 2013.

[14] V. Emamian, P. Anghel, and M. Kaveh, "Multi-user spatial diversity in a shadow-fading environment," in Proc. IEEE VTCFall, Sep. 2002, pp. 573-576.

[15] H. Holma and A. Toskala, LTE for UMTS: Evolution to LTEAdvanced, Wiley, 2nd edition edition, 2011.

[16] I. S. Gradshteyn and I. M. Ryzhik, Table of Integrals, Series, and Products, Academic Press, 7th edition, 2007.

[17] A. Goldsmith, Wireless Communications, Cambridge University Press, 2005.

[18] V. K. N. Lau and M. Jiang, "Downlink scheduling and rate adaptation design of multi-user, multiple-antenna base station with imperfect CSIT," in Proc. IEEE GLOBECOM, Dec. 2005. 